\title{
Recent hard probe measurements from STAR
}

\author{
Fuqiang Wang ${ }^{1, a}$ (for the STAR Collaboration) \\ ${ }^{1}$ Department of Physics and Astronomy, Purdue University, West Lafayette, IN 47907, USA
}

\begin{abstract}
High momentum transfer processes can be reliably calculated by perturbative quantum chromodynamics (pQCD). Products of those hard processes, high transverse momentum particles, jets, and heavy quarks (hard probes), interact with the quark-gluon plasma (QGP) created in relativistic heavy-ion collisions. Properties of the QGP can be studied by comparing hard probe measurements in heavy-ion collisions to those in $p+p$ collisions or from pQCD calculations. I will present recent results from STAR on hard probe measurements, including a wide range of observables: heavy flavor, dijet transverse momentum imbalance, hadron-jet correlations, $\gamma$-jet correlations, and dihadron correlations. I will discuss what we have learned from these measurements.
\end{abstract}

\section{Introduction}

The primordial universe was immersed by the most elementary particles: quarks, gluons, leptons, and photons. This extremely high energy density state-the quark-gluon plasma (QGP)-is created in today's laboratory by relativistic heavy-ion collisions [1-3]. The question scientists want to answer, among others, is how the fundamental theory of quantum chromodynamics (QCD) behaves in the high density medium, such as the QGP, and what novel phenomena may emerge. Two main ways to study the QGP are to measure how it dissembles, much like how the universe expanded and cooled down, and how energetic quarks and gluons (hard probes) interact with it and fragment into showers of collimated hadrons (jets). Hard probes are a valuable tool to study the QGP in nuclear (AA) collisions because their initial production can be reliably calculated by perturbative QCD (pQCD) or measured in proton+proton (pp) collisions. The effect of their interactions with the QGP can, therefore, be studied by comparing AA collisions to pp collisions and/or pQCD calculations.

This proceedings article reviews hard probe results from the STAR experiment at RHIC. In particular, the following hard probe results are reviewed: heavy quark production and collective flow, dijet transverse momentum $\left(p_{T}\right)$ imbalance, hadron-jet correlations, $\gamma$-hadron correlations, and dihadron correlations.

\section{Heavy flavor probe}

One central question is how the QGP quickly thermalizes from the initial non-equilibrium state of heavy-ion collisions. Heavy quarks, referred to as heavy flavor (HF), are a sensitive probe to this question because they, being heavy, do not thermalize easily.

a e-mail: fqwang@purdue.edu 
Figure 1, left panel, shows the nuclear modification factor, $R_{A A}$, the ratio of production rate of $D^{0}$ mesons (containing a heavy flavor charm quark) in $\mathrm{Au}+\mathrm{Au}$ collisions to that in pp collisions properly normalized by the number of binary nucleon-nucleon collisions [4]. $D^{0}$ production is strongly suppressed at large $p_{T}$, a result of charm quark energy loss due to interactions with the QGP.
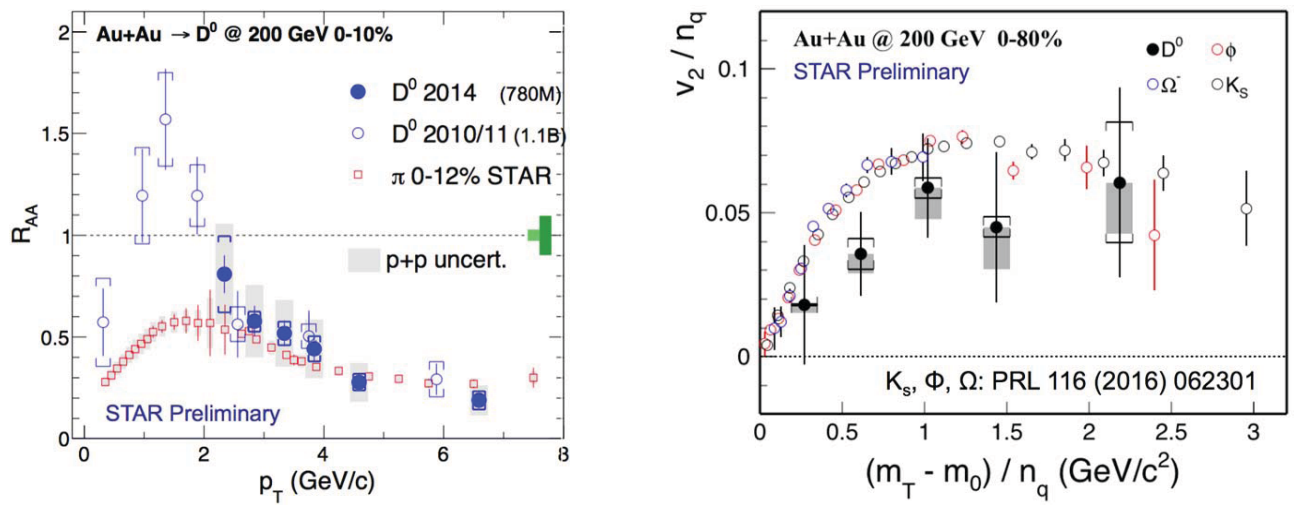

Figure 1. (Color online) Nuclear modification factor $R_{A A}$ [4] (left) and elliptic anisotropy (right) [5] of $D^{0}$ mesons in $\mathrm{Au}+\mathrm{Au}$ collisions, compared to those of other particles.

Figure 1, right panel, shows the elliptic azimuthal anisotropy of $D^{0}$ mesons [5], compared to that of other hadrons. This azimuthal anisotropy is generated by interactions of charm quarks with the QGP. Large anisotropy values are generally taken as evidence of strong interactions and low viscosity hydrodynamics. The comparable magnitude of $D^{0}$ anisotropy to that of light hadrons' would suggest that heavy quarks are as thermalized as light quarks, which implies that the QGP is highly thermalized. Recent transport studies [6] and other approaches suggest that hydrodynamics may not be a unique explanation for the anisotropy.

\section{Dijet transverse momentum imbalance}

Jets are produced back-to-back in azimuth by hard scatterings of partons, initially moving, in leading order QCD, along the longitudinal directions. Higher order effects of QCD, e.g. nucleon Fermi motion in transverse direction inside the nucleus and initial-state gluon radiation of partons in the nucleons, render a non-strict back-to-back balance of dijet $p_{T}$. Interactions with the QGP produce additional $p_{T}$ imbalance between leading and subleading jets of the dijet system. Comparison of the $p_{T}$ imbalance, quantified by the $A_{j} \equiv \frac{p_{T}^{\text {lead }}-p_{T}^{\text {sublead }}}{p_{T}^{\text {lead }}+p_{T}^{\text {sublead }}}$ variable, between $A A$ and pp collisions will reveal the strength of jet-medium interactions.

Figure 2 shows the $A_{j}$ distributions in AA and pp collisions [7] by the red solid and open symbols, respectively. The $A_{j}$ distribution in AA collisions is shifted to larger values than that in pp collisions. This shift is the effect of additional $p_{T}$ imbalance due to jet interactions with the QGP.

Some words are in order here on the details of jets measured in experiments. Jets are reconstructed by clustering final-state particles in a cone of given size $R=\sqrt{\Delta \eta^{2}+\Delta \phi^{2}}$ where $\Delta \eta$ is spread in pseudorapidity $(\eta)$ and $\Delta \phi$ is that in azimuth. There are several jet reconstruction algorithms; the jet results in this article are obtained by the anti- $k_{T}$ algorithm which emphasizes high- $p_{T}$ particles [8]. In Fig. $2, R=0.4$ is used. Not all energy of the initial parton is reconstructed within the finite size 


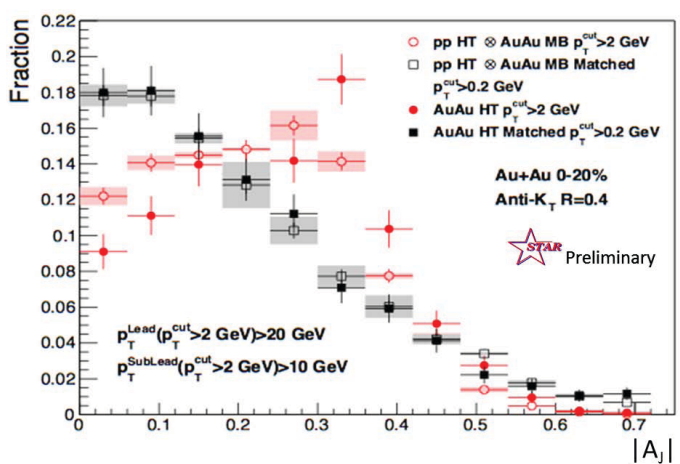

Figure 2. (Color online) Dijet $p_{T}$ imbalance in central AA and pp collisions [7]. Jets are reconstructed by the anti- $k_{T}$ algorithm with $R=0.4$, and particle $p_{T}>2 \mathrm{GeV} / c$ (red points). Results with the same dijets but including $p_{T}>0.2 \mathrm{GeV} / c$ particles in jet energy are shown in black.

because of out-of-cone radiation. In addition, a lower $p_{T}>2 \mathrm{GeV} / c$ cutoff is imposed in order to reduce background. The broadened $A_{j}$ distribution in AA indicates energy loss (energy is shifted from high $p_{T}$ to low $p_{T}$ ) and/or broadening (energy is shifted out of cone).

Once jets are reconstructed with the fragment $p_{T}>2 \mathrm{GeV} / c$ cut, this $p_{T}$ cut can be relaxed or removed to include jet energies residing in low- $p_{T}$ particles. The black filled and open points in Fig. 2 show the $A_{j}$ results with a relaxed $p_{T}>0.2 \mathrm{GeV} / c$ (dictated by the detector aperture) for AA and pp collisions, respectively. The two distributions are consistent with each other, suggesting that jetmedium interactions result in jet softening, but not broadening beyond $R=0.4$. For smaller $R$ cone size used in jet reconstruction, however, dijet $A_{j}$ distributions are found not to match between AA and pp (broader in $A A$ than pp) even with $p_{T}>0.2 \mathrm{GeV} / c$. This indicates that jet-medium interactions do result in jet broadening, besides softening, but only within $R=0.4$. Note that the dijets shown in Fig. 2 are required to satisfy $p_{T}^{\text {lead }}>20 \mathrm{GeV} / c$ and $p_{T}^{\text {sublead }}>10 \mathrm{GeV} / c$, relatively large for RHIC energy of $200 \mathrm{GeV}$. At the LHC it is found that jets are broadened to significantly larger $R$ at low $p_{T}[9]$.

\section{Hadron-jet correlations}

Jet-medium interactions can be studied in more detail by hadron-jet correlations where the hadron, called the trigger, is required to be at high $p_{T}$. Because of energy loss and the expeditiously dropping jet production with increasing $p_{T}$, those high- $p_{T}$ trigger hadrons are predominantly produced by hard scattering near the "cylindrical" surface of the collision zone and directed outward. The back-to-back jet partners are mostly directed inward, having to traverse the entire size of the collision zone.

Figure 3 shows that the charged hadron jet $p_{T}$ spectra for $R=0.3$ and $R=0.5$ [10]. The jet area $A_{\text {jet }}$ is required to be larger than 0.20 and 0.65 , respectively, to reduce fake jets. All charged particles of $p_{T}>0.2 \mathrm{GeV} / c$ are include in jet reconstruction (anti- $k_{T}$ ). In heavy-ion collisions, with essentially no $p_{T}$ cut imposed on particles, fake jet rate is large. In this analysis fake jets are reconstructed by a mixed-event technique, where one and only one track is sampled from each event of similar characteristics (centrality, event plane, etc.). The mixed-event technique yields a good description of fake jets in kinematic ranges where all reconstructed jets are known to be fake. The mixed-event fake jets have been subtracted for the spectra in Fig. 3. In addition, the jet energy has been corrected for underlying event contribution by subtracting $\rho A_{\text {jet }}$ where $\rho$ is the medium area density of the background particle energy, and unfolded for underlying event background fluctuations and particle momentum resolution. 

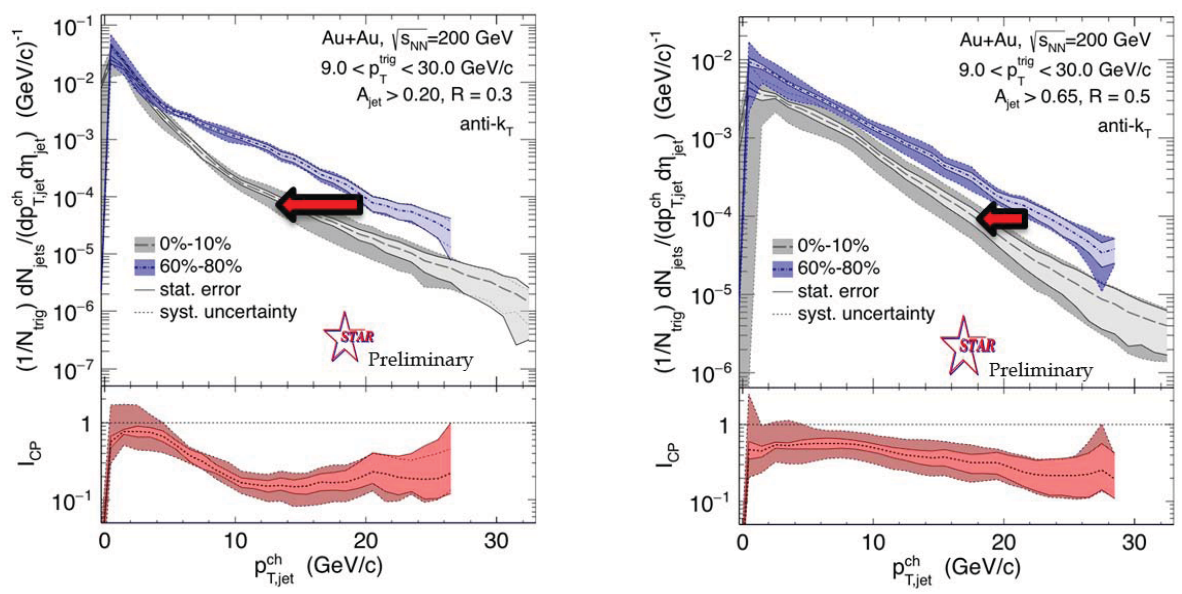

Figure 3. (Color online) Hadron-jet correlations [10]: recoil jet $p_{T}$ spectra in peripheral and central $\mathrm{Au}+\mathrm{Au}$ collisions for $R=0.3$ and $R=0.5$ (upper) and $I_{A A}$, ratio of jet spectra in central to peripheral collisions (lower).

In Fig. 3, the trigger hadron $p_{T}$ range is $9-30 \mathrm{GeV} / c$. Both the peripheral and central jet spectra are shown. The spectra ratio of central to peripheral collisions is shown in the lower panels of Fig. 3 . The central spectrum is suppressed at large $p_{T}$ indicating jet energy loss in medium. The suppression can be viewed as the result of a horizontal shift to the left of the spectra from peripheral to central collisions. The shift indicates the amount of energy loss. The shift is smaller for larger $R$ at RHIC, and for $R=0.5$, is smaller at RHIC than LHC [11].

\section{$5 \gamma$-hadron correlations}

Reconstructed jet energy does not precisely reflect the parent parton energy or the hard scattering momentum transfer. Jet studies always involve data-theory comparisons where jets are reconstructed in the exactly same way in real data and theory calculations. Gamma-jet correlations, on the other hand, offer a direct measure of the hadron scattering momentum transfer and a gauge of the recoil jet energy. Gamma-hadron correlations, a prestep toward the golden $\gamma$-jet measurements, have been widely studied.

Figure 4 shows $I_{A A}$, the ratio of jet fragmentation functions in AA collisions to pp collisions, where $z_{T} \equiv p_{T}^{\text {hadron }} / p_{T}^{\gamma}$ [12]. The $\gamma$-hadron data are compared to the $\pi^{0}$-hadron correlation data where the $z_{T} \equiv p_{T}^{\text {hadron }} / p_{T}^{\pi^{0}}$ for the latter. The trigger $p_{T}^{\gamma}$ and $p_{T}^{\pi^{0}}$ are restricted within $12-20 \mathrm{GeV} / c$ and the hadron $p_{T}$ is required to be larger than $1.2 \mathrm{GeV} / c$. The recoil jets from $\pi^{0}$ or $\gamma$ are both strongly suppressed. The suppression is less significant at low $z_{T}$ (low associated hadron $p_{T}$ ). With the current statistical precision, the $\gamma$-hadron and $\pi^{0}$-hadron correlation data are compatible. Note, however, that (1) the energies of the recoil jets in the two cases are different because the $\pi^{0}$ takes only portion of the jet energy while the $\gamma$ closely reflects the recoil jet energy, thus the $z_{T}$ definitions are different for these two measurements; (2) the $\pi^{0}$-hadron correlations are biased to surface hard scatterings and the $\gamma$-hadron correlations are unbiased (except for possible bias introduced by the $p_{T}^{\text {hadron }}>1.2 \mathrm{GeV} / c$ cut); and (3) the recoil jet from the $\gamma$ is mostly quark jet and that recoil the $\pi^{0}$ is mostly gluon jet. 


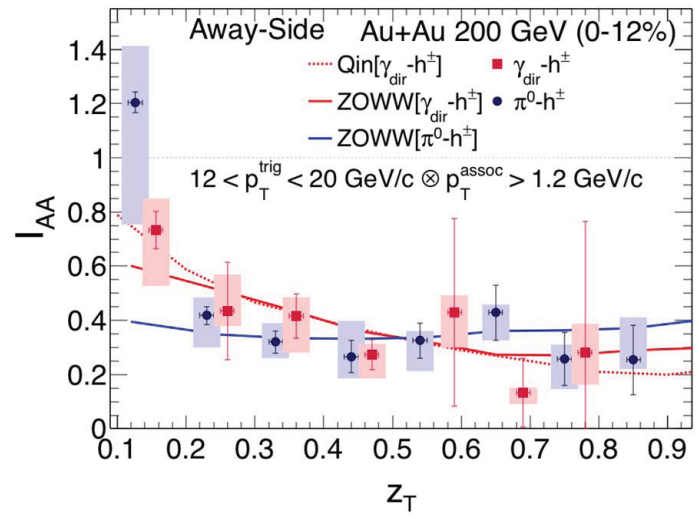

Figure 4. (Color online) $\gamma$-hadron correlations [12]: ratio of the recoil jet "fragmentation" function in $\mathrm{Au}+\mathrm{Au}$ collisions to that in pp collisions. The data are compared to those from $\pi^{0}$-hadron correlations and theoretical calculations.

Figure 4 also shows comparisons to theoretical calculations. The calculations and data are consistent within uncertainties.

\section{Dihadron correlations}

Jet-medium interactions shift energy from high- $p_{T}$ particles to low- $p_{T}$ particles. The bulk of jetmedium interaction effects is contained in low- $p_{T}$ particles. Jet-correlated low $p_{T}$ have been most difficult to measure because of the overwhelming background from the underlying event. The azimuthal distribution shape of this background is "arbitrary" because of the possible azimuthal harmonics of all orders due to initial state geometry fluctuations. Subtraction of this background suffers from large systematic uncertainties.

A novel method has been recently devised by requiring large $p_{T}$ recoil from a relatively high$p_{T}$ trigger particle and subtracting anisotropic underlying event background using data itself [13]. Figure 5 shows the Gaussian width of the away-side correlation peak from this method as a function of centrality [13]. The width increases with centrality; the increase can be due to the increasing nuclear $k_{T}$ broadening with centrality, broadening due to jet-medium interactions, or both. To shed more light, Fig. 5 right panel shows the widths in peripheral and central collisions and the quadratic difference of the widths as a function of $p_{T}$. Because the change in the nuclear $k_{T}$ effect from peripheral to central collisions does not depend on hadron $p_{T}$, the increase of the width difference with $p_{T}$ indicates the effect of jet-medium interactions; the effect seems larger for high $p_{T}$ correlated particles.

\section{Summary}

STAR has made a variety of hard probe measurements. All hard probes are suppressed in heavyion collisions compared to pp collisions and pQCD calculations. Lost energy by hard probes are shifted to lower $p_{T}$ and larger angles. Heavy quarks flow, almost as strongly as light quarks. These measurements show strong medium effects; more quantitative knowledge about the medium requires rigorous theory and model comparisons to the data.

\section{References}

[1] K. Adcox et al. (PHENIX Collaboration), Nucl.Phys. A757, 184 (2005), nucl-ex/0410003

[2] J. Adams et al. (STAR Collaboration), Nucl.Phys. A757, 102 (2005), nucl-ex/0501009 

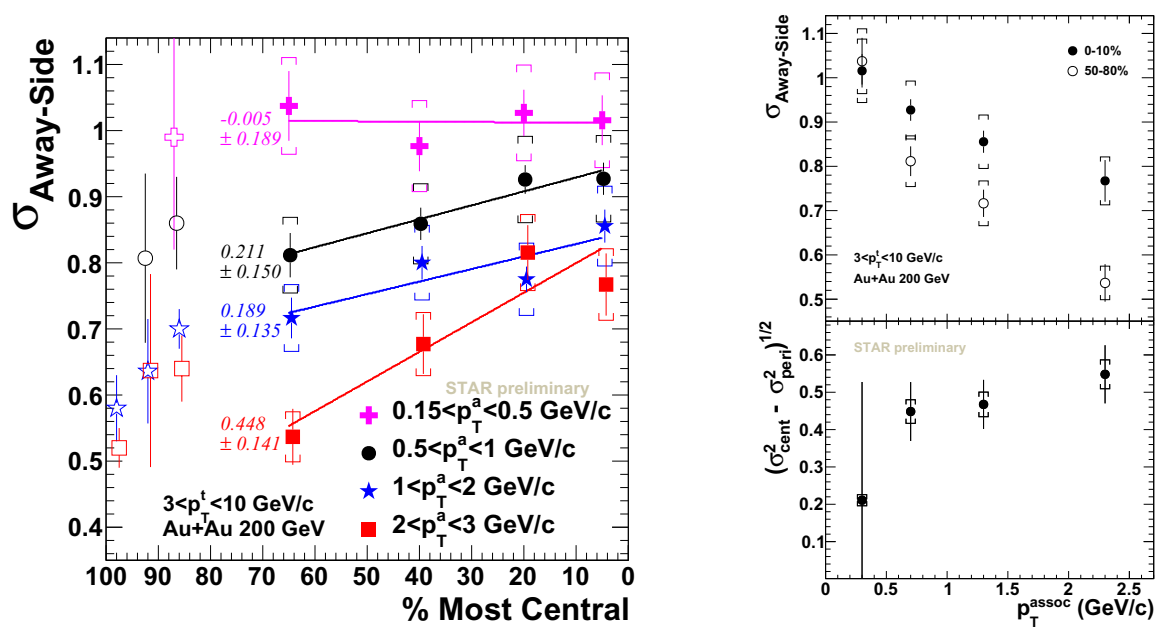

Figure 5. (left) Away-side dihadron correlation peak width as a function of centrality for various associated particle $p_{T}$ ranges. (right) Away-side peak width in peripheral and central collisions (upper) and the quadratic difference of the widths (lower) as a function of associated particle $p_{T}$.

[3] B. Muller, J. Schukraft, B. Wyslouch, Ann.Rev.Nucl.Part.Sci. 62, 361 (2012), 1202 . 3233

[4] G. Xie (STAR), Nucl. Phys. A956, 473 (2016), 1601.00695

[5] M.R. Lomnitz (STAR), Nucl. Phys. A956, 256 (2016), 1601.00743

[6] L. He, T. Edmonds, Z.W. Lin, F. Liu, D. Molnar, F. Wang, Phys. Lett. B753, 506 (2016), 1502.05572

[7] L. Adamczyk et al. (STAR) (2016), 1609. 03878

[8] M. Cacciari, G.P. Salam, G. Soyez, JHEP 0804, 063 (2008), 0802 . 1189

[9] S. Chatrchyan et al. (CMS), Phys. Rev. C84, 024906 (2011), 1102 . 1957

[10] P.M. Jacobs, A. Schmah (STAR), Nucl. Phys. A956, 641 (2016), 1512.08784

[11] J. Adam et al. (ALICE), JHEP 09, 170 (2015), 1506.03984

[12] N.R. Sahoo (STAR), Nucl. Phys. A956, 621 (2016), 1512.08782

[13] K. Jiang (STAR Collaboration), J. Phys. Conf. Ser. 636, 012011 (2015) 